\title{
Symmetry-protected topological photonic crystal in three dimensions
}

\author{
Ling Lu ${ }^{1 \star i}$, Chen Fang ${ }^{1 \uparrow *}$, Liang Fu ${ }^{1 \star}$, Steven G. Johnson ${ }^{2}$, John D. Joannopoulos ${ }^{1}$ and Marin Soljačić ${ }^{1}$
}

Topology of electron wavefunctions was first introduced to characterize the quantum Hall states in two dimensions discovered in 1980 (ref. 1). Over the past decade, it has been recognized that symmetry plays a crucial role in the classification of topological phases, leading to the broad notion of symmetry-protected topological phases ${ }^{2}$. As a primary example, topological insulators ${ }^{3,4}$ are distinguished from normal insulators in the presence of time-reversal symmetry $(\mathcal{T})$. A three-dimensional (3D) topological insulator ${ }^{3-6}$ exhibits an odd number of protected surface Dirac cones, a unique property that cannot be realized in any 2D systems. Importantly, the existence of topological insulators requires Kramers' degeneracy in spin-orbit coupled electronic materials; this forbids any direct analogue in boson systems ${ }^{7}$. In this report, we discover a 3D topological photonic crystal phase hosting a single surface Dirac cone, which is protected by a crystal symmetry $^{8-10}$-the nonsymmorphic glide reflection ${ }^{11-13}$ rather than $\mathcal{T}$. Such a gapless surface state is fully robust against random disorder of any type $\mathrm{e}^{14,15}$. This bosonic topological band structure is achieved by applying alternating magnetization to gap out the 3D 'generalized Dirac points' discovered in the bulk of our crystal. The $Z_{2}$ bulk invariant is characterized through the evolution of Wannier centres ${ }^{16}$. Our proposalreadily realizable using ferrimagnetic materials at microwave frequencies ${ }^{17,18}$-expands the scope of $3 \mathrm{D}$ topological materials from fermions to bosons.

Unlike in Fermi systems, achieving a single Dirac cone in boson systems requires $\mathcal{T}$ breaking. This is because the $\mathcal{T}$ operator acts differently on bosons and fermions: $\mathcal{T}_{\mathrm{f}}^{2}=-1$ for fermions with halfinteger spins and $\mathcal{T}_{\mathrm{b}}^{2}=1$ for bosons with integer spins. As a result, $\mathcal{T}_{\mathrm{b}}$ is not compatible with the Hamiltonian of a single Dirac cone, whereas $\mathcal{T}_{\mathrm{f}}$ is (see Supplementary Information). Instead of $\mathcal{T}_{\mathrm{f}}$, the Dirac point degeneracy in our photonic crystal is protected by a glide reflection, which ensures an odd number of band crossings on two high-symmetry lines in the surface Brillouin zone (BZ; refs 12,13). This $3 \mathrm{D}$ topological photonic crystal is a material realization of the recently proposed nonsymmorphic topological phase $^{11-13,19}$ and can be regarded as a bosonic analogue of both the 3D topological insulator ${ }^{3-5}$ (in terms of the single surface Dirac cone) and the topological crystalline insulators ${ }^{8-10}$ (in terms of the crystal-symmetry protection) in electronic systems.

Our starting point is a photonic crystal having a body-centredcubic (bcc) unit cell which contains four identical dielectric rods, illustrated with different colours for clarity in Fig. 1b. This crystal belongs to the nonsymmorphic space group No. $230(\operatorname{Ia} \overline{3} d)$ that contains glide reflections and inversion. Interestingly, such a triply periodic structure self-assembles as disclination-line networks in the first blue phase of liquid crystals ${ }^{20}$, denoted as BP I. Here, the dielectric constant $(\varepsilon)$ of the rods is 11 and the radius is $0.13 a$, where $a$ is the length of the cubic cell. In Fig. 1a, the photonic band structure of BP I shows a four-fold degenerate point at the $\mathrm{P}$ momentum, dispersing linearly in all directions of the $3 \mathrm{D}$ momentum space. Unlike a regular 3D Dirac point ${ }^{21,22}$-a four-fold degeneracy point which splits into two sets of doublet bands along any direction-our four-fold degeneracy here splits into four bands along a generic direction. In Fig. 1a, this splitting is not obvious, because most dispersions still remain doubly degenerate along highsymmetry momentum lines. However, it is clear that the third and fourth bands split along $\mathrm{P}-\Gamma$ and the first and second bands split along $\mathrm{P}-H$. We name this type of degeneracy ${ }^{23}$ a $3 \mathrm{D}$ 'generalized Dirac point' (GDP). We note that there are two non-equivalent $\mathrm{P}$ points in the $3 \mathrm{D}$ bcc $\mathrm{BZ}$ related by inversion. Interestingly, the two GDPs (at $\pm P$ ) are the only band touchings between band 1,2 and 3,4 . When the space group is perturbed, the GDPs could turn into line nodes, Weyl points ${ }^{24,25}$ or open bandgaps. Detailed studies of GDPs will be presented in another paper.

In symmorphic space groups, where the point groups decouple from lattice translations, the highest dimension of group representation is three. The four-fold band degeneracies of the GDPs are hence the consequence of the nonsymmorphic symmetries of glide reflections and screw rotations in BPI. A nonsymmorphic symmetry is in general composed of a point group (mirror or rotation) followed by a fractional lattice translation, where neither of the two is a symmetry of the system. The important feature of a nonsymmorphic space group is the extra band degeneracies at the $\mathrm{BZ}$ boundaries ${ }^{26-31}$. Because the screw rotations cannot be preserved on a planar surface, we focus on the glide reflections to obtain protected surface states. Shown in Fig. 1b, the (001) surface has two glide reflections: $G_{x}=\left\{M_{x} \mid(a / 2) \hat{x}-(a / 2) \hat{y}\right\}$ and $G_{y}=\left\{M_{y} \mid(a / 2) \hat{x}\right\}$. The inversion centre is at the origin of the unit cell inside the glide plane of $G_{y}$. The top-view schematic illustrates the relations between the four rods under the two glide reflections. The (001) surface BZ is plotted in Fig. 1c.

A glide reflection ensures a linear point degeneracy along each glide-reflection-invariant momentum line. To see this, we study the Bloch states on the $G_{y}$-invariant lines of $\mathrm{X}^{\prime}-\mathrm{X}$ and $\mathrm{M}^{\prime}-\mathrm{M}$ shown dashed in the (001) surface BZ in the right-hand panel of Fig. 2a. A Bloch state with momentum $\left(k_{x}, k_{y}\right)$ is mapped to another state with momentum $\left(k_{x},-k_{y}\right)$ under $G_{y}$, so for any state along these two lines with $k_{y}=0$ and $k_{y}=\pi / a$, its momentum is invariant under $G_{y}$. This means the eigenvalues of $G_{y}\left(g_{y}\right)$ are good quantum numbers

${ }^{1}$ Department of Physics, Massachusetts Institute of Technology, Cambridge, Massachusetts 02139, USA. ${ }^{2}$ Department of Mathematics, Massachusetts Institute of Technology, Cambridge, Massachusetts 02139, USA. †These authors contributed equally to this work. Fresent address: Beijing National Laboratory for Condensed Matter Physics, and Institute of Physics, Chinese Academy of Sciences, Beijing 100190, China. *e-mail: linglu@mit.edu; liangfu@mit.edu 
a

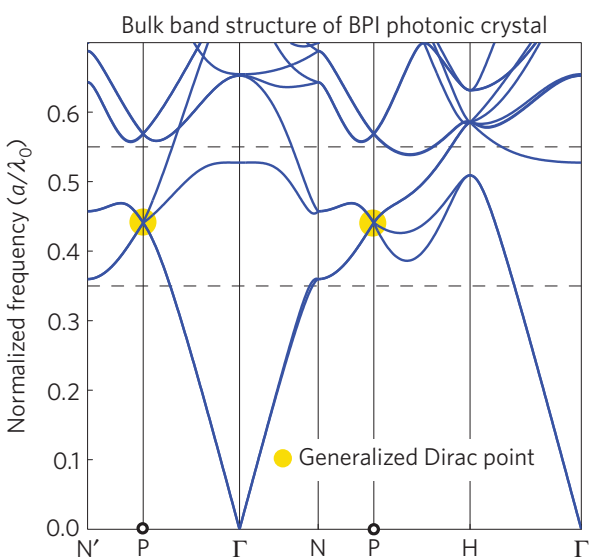

b

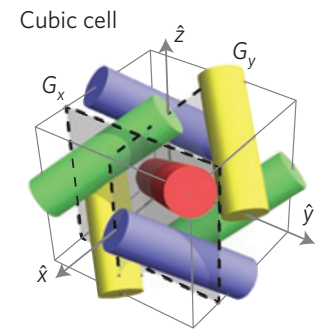

d

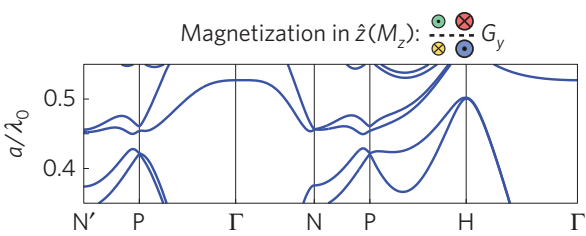

(001) view

schematic

$G_{x}$

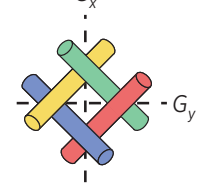

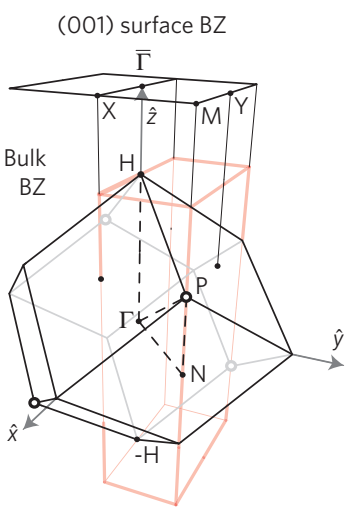

Figure 1 | BP I photonic crystal. a, Bulk band structure of the BP I photonic crystal in the bcc lattice. The GDP is between the first four bands. b, Cubic unit cell of length a consisting of four identical dielectric rods oriented along the bcc lattice vectors of (111) (red), (111) (yellow), (1111) (blue) and (1)11) (green). The rods go through $(0,0,0) a,(0,0.5,0) a,(0.5,0,0) a$ and $(0,0,0.5) a$, respectively. There are two glide reflection planes $\left(G_{x}\right.$ and $\left.G_{y}\right)$ in the structure,

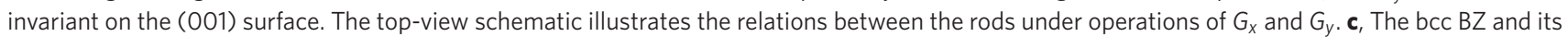
projection onto the (001) surface BZ. The transparent red box outlines the volume in the bulk BZ that projects to half of the surface BZ. d, Bulk band structure showing that the GDP opens when magnetization is applied on the rods without breaking $G_{y}$.

for the Bloch states on these two lines. Because $G_{y}{ }^{2}=\{1 \mid a \hat{x}\}$, $g_{y}\left(k_{x}\right)= \pm \mathrm{e}^{-i k_{x} a / 2}\left[g_{y}^{2}\left(k_{x}\right)=\mathrm{e}^{-i k_{x} a}\right]$, which is dependent on $k_{x}$. The two branches of glide-reflection eigenvalues always differ by a minus sign, and evolve into each other after a $2 \pi$ transportation along the $G_{y}$-invariant lines owing to the fact that $g_{y}\left(k_{x}\right)=-g_{y}\left(k_{x}+(2 \pi / a)\right)$. As a result, the corresponding wavefunctions of the two branches have the same winding as their eigenvalues-a unique property of the half-lattice translation in glide reflections. Consequently, the two frequency eigenvalues of the two Bloch modes also switch values after transporting a period along the invariant momentum lines, illustrated in Fig. 2a. Assume the frequencies of the two modes are $\omega^{+}$and $\omega^{-}$at an arbitrary $k_{x}$ point (say $k_{x} a=0$ ). The frequency dispersions switch their values at $k a=2 \pi$. This switch ensures a crossing point (red dot) on $\mathrm{X}^{\prime}-\mathrm{X}$ and $\mathrm{M}^{\prime}-\mathrm{M}$, respectively. We argue that these two protected double degeneracies give a $Z_{2}$ classification of the surface states ${ }^{12}$. Illustrated in the middle panel of Fig. 2a, there are two topologically inequivalent ways for these two point degeneracies to connect. The gapless connection is a signature of the topologically nontrivial surface state protected by $G_{y}$.

We now break $\mathcal{T}$ in the BPI photonic crystal to open the bulk bandgap without breaking $G_{y}$. Shown in Fig. 1d, the GDP at the $\mathrm{P}$ point lifts up into a bandgap when we apply alternating magnetizations on the rods along $\hat{z}$. These magnetizations induce off-diagonal imaginary parts in the dielectric constant $(\varepsilon)$ of materials with a gyroelectric response ${ }^{32}$. (Ferrimagnetic materials with a gyromagnetic response ${ }^{17}$ give the same results in Supplementary Information.) Here $\mu=1$ and

$$
\varepsilon=\left(\begin{array}{ccc}
\varepsilon_{\|} & \kappa & 0 \\
-\kappa & \varepsilon_{\|} & 0 \\
0 & 0 & \varepsilon_{z z}
\end{array}\right)
$$

where $\varepsilon_{z z}=11, \varepsilon_{\|}^{2}-|\kappa|^{2}=\varepsilon_{z z}^{2} \quad$ (ref. 24) and $\kappa$ is a non-zero imaginary number when the magnetization $\left(\mathrm{M}_{z}\right)$ is present. In Fig. $1 \mathrm{~d}, \kappa=-10 i,-5 i,+5 i,+10 i$ for the red, yellow, green and blue rods, respectively. This configuration preserves $G_{y}$, because magnetization (magnetic field) flips sign under a mirror (reflection) operation. The $2 \mathrm{D}$ plane group of the resulting (001) surface is $p g$.

The (001) surface state, plotted in Fig. 2b, has a single Dirac cone at the $\mathrm{L}$ point on the $\mathrm{M}^{\prime}-\mathrm{M}$ line, consistent with the glide-reflection degeneracy in Fig. 2a. By varying the magnetization or rod radius without breaking $G_{y}$, the Dirac point L moves along the $G_{y}$-invariant line $\mathrm{M}^{\prime}-\mathrm{M}$. This single Dirac cone at $\mathrm{L}$ is connected gaplessly with the bulk bands across the bandgap. In Fig. 2c, we restore $G_{x}$ to coexist with $G_{y}$ by doubling the magnetization amplitude of the green and yellow rods $(|\kappa|$ from 5 to 10$)$. The surface plane group becomes $p 2 g g$. Owing to this extra glide-reflection plane through the $\mathrm{Y}$ point, the surface Dirac cone is then pinned at $\mathrm{Y}$ on $\mathrm{M}^{\prime}-\mathrm{M}$. If we break both glide symmetries by de-magnetizing the yellow rod, both glide planes of $G_{x}$ and $G_{y}$ are broken and the surface plane group reduces to $p 1$. The surface Dirac cone is now gapped, as shown in Fig. 2d. This demonstrates that the gapless surface states are indeed protected by the glide reflection.

The principle of bulk-edge correspondence says that the surface state is a holographic representation of the bulk topology. We demonstrate this correspondence between the surface states in Fig. 2 and the 'hybrid Wannier centres' 16 of the bulk bands below the bandgap computed in Fig. 3. This approach is also known as the Wilson loops $\mathrm{s}^{33,34}$. The hybrid Wannier function of each band is a spatially localized wavefunction along $z$, obtained from Fourier transforming the Bloch wavefunctions with respect to $k_{z}$ while keeping the other two surface momenta. The $z$-position expectation values of the hybrid Wannier wavefunctions-that is, the hybrid Wannier centres, are equivalent to the Berry phases of the bulk bands below the gap along a loop in $\hat{z}$ in the bulk BZ. In our bcc lattice, this non-contractable loop of length $4 \pi / a$ (instead of $2 \pi / a)$ is the vector connecting $H$ and $-H$ in Fig. 1c. So the hybrid Wannier centre is well defined up to a lattice period of $a / 2$ (instead of $a$ ) in $\hat{z}$ and, similarly, the Berry phase has a $2 \pi$ phase ambiguity. The calculations of the Berry phases are detailed in the Supplementary Information.

In the surface BZ, a gapless spectrum of Wannier centres (Berry phases) indicates a nontrivial bulk topology and a gapless surface state. In contrast, a gapped spectrum represents a trivial bulk topology and the absence of gapless surface states. This can be understood by the following intuitive arguments. If there is a full gap in the spectrum of Wannier centres, then there is a certain position in $z$ where no state is localized. Terminating the bulk at that plane results in a surface without surface states-the trivial surface states. On the other hand, if the Wannier centre plot is gapless, then for any surface termination there must be a localized surface state at some surface momentum. The surface is hence gapless for terminations at arbitrary $z$-a nontrivial surface. In Fig. 3, we plot the Wannier centres of the two lowest bands along the closed loop of 

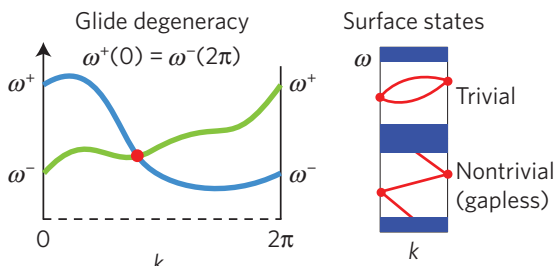

b

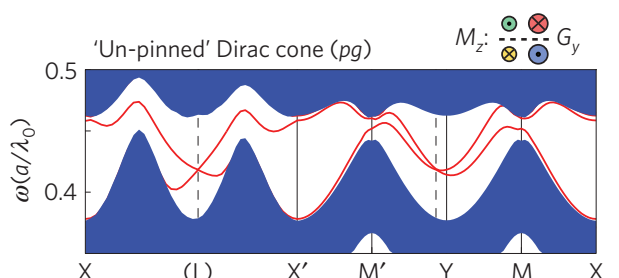

(001) surface BZ

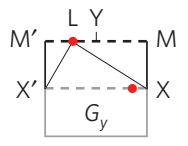

Surface states

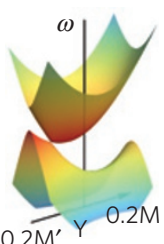

$G_{x}$
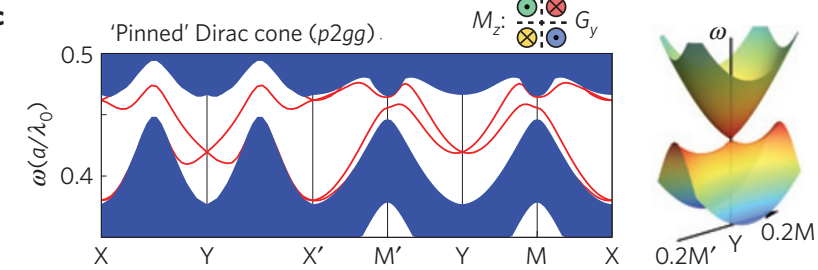

d
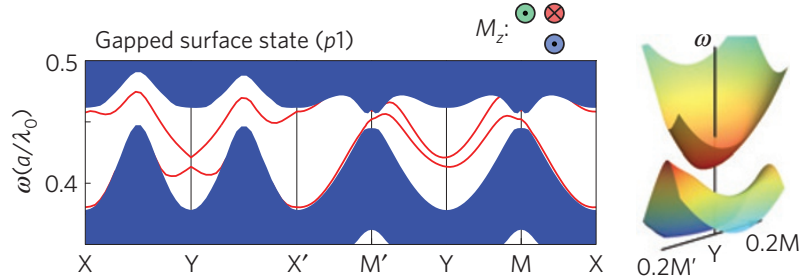

Figure 2 | The (001) surface states after breaking $\mathcal{T}$. The surface and bulk dispersions are plotted in red and blue, respectively, along the surface momentum lines that go through the surface Dirac point twice to show its anisotropy. More surface data are plotted in Supplementary Fig. 1. a, Illustration of the two crossing points (double degeneracies) on the $G_{y}$-invariant lines of $M^{\prime}-M$ and $X^{\prime}-X$ (dotted) in the surface BZ. Consequently, the surface states

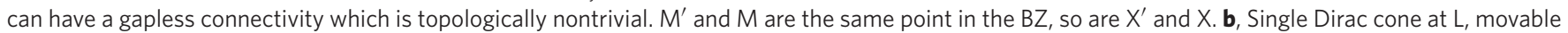
along the $M^{\prime}-M$ line protected by $G_{y}$. c, Single Dirac cone in $\mathbf{b}$, pinned at the $Y$ point owing to the coexistence of $G_{x}$ and $G_{y}$. $\mathbf{d}$, Surface states in $\mathbf{b}, \mathbf{c}$ gapped by breaking the glide-reflection symmetries. For all above surface calculations, a perfect metallic boundary is placed on top, at the centre of the cubic cell, on the (001) surface with an air gap spacing of 0.5 from the photonic crystal surface. The 3D plots of the surface dispersions are plotted on the right to

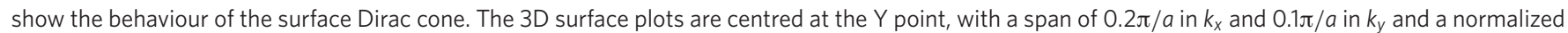
frequency range between 0.41 and 0.43 .

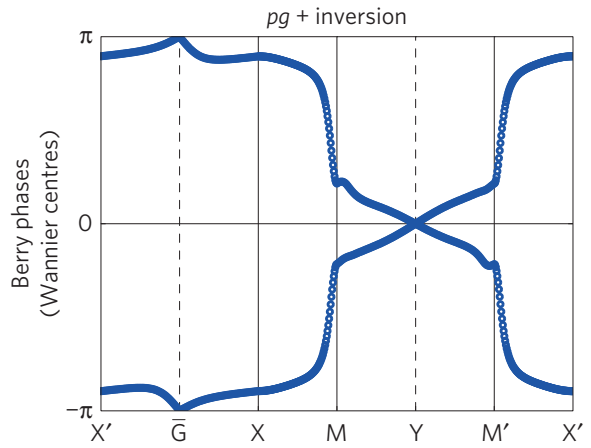

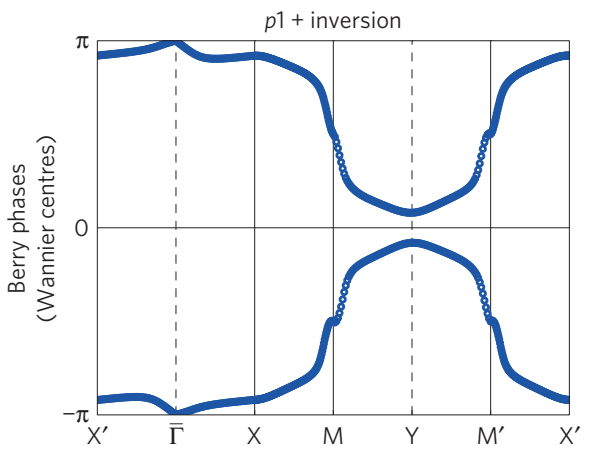

Figure $\mathbf{3}$ | Hybrid Wannier centres in the surface BZ indicating bulk topologies and the connections of the surface states. a, Gapless hybrid Wannier centres corresponding to the nontrivial surface states $(p g)$ in Fig. 2b. b. Gapped hybrid Wannier centres corresponding to the trivial surface states ( $p 1$ ) in Fig. 2d. The hybrid Wannier centres corresponding to the $p 2 g g$ surface in Fig. 2c are plotted in the Supplementary Information.

$\mathrm{X}^{\prime}-\mathrm{X}-\mathrm{M}-\mathrm{M}^{\prime}-\mathrm{X}^{\prime}$ in the surface $\mathrm{BZ}$. Figure $3 \mathrm{a}$ depicts the hybrid Wannier centres calculated for the bulk bands in Fig. 1d, whose surface state is shown in Fig. 2b. Similarly, the hybrid Wannier centres in Fig. 3 b correspond to the surface states shown in Fig. 2 d. The Wannier centres are gapless in Fig. 3a, consistent with the existence of the gapless single surface Dirac cone in Fig. 2b. In comparison, the Wannier centres in Fig. $3 \mathrm{~b}$ are gapped, also consistent with the absence of topological surface states in Fig. $2 \mathrm{~d}$. These data confirm the bulk-edge correspondence that the Wannier centres for all bulk bands below the bandgap are homotopic to the surface band structure of a semi-infinite system with one open surface.

Single-Dirac-cone surface states are fully robust and do not localize under arbitrary random disorders on the surface. This has been discussed in 3D topological insulators, where the surface states remain delocalized under random impurities of any type $e^{14,15}$, assuming that spontaneous symmetry breaking does not occur. In our case, although individual defects break the glide reflection, their ensemble average does not. Intuitively, if one local disorder generates a positive Dirac mass term within a region on the surface, there must be a neighbouring region where the mass term is negative. A chiral edge mode exists along the edge between two regions with opposite masses, similar to the photonic oneway edge states ${ }^{17,32}$, analogous to the quantum Hall effect. In the presence of strong disorder, these chiral edge modes percolate the surface and the surface states remain delocalized. The surface with a strong random disorder can be mapped to the electronic states at the critical point of a quantum Hall plateau transition, where chiral edge modes between regions of different Landaulevel filling factors percolate. The transmission rate of light on the surface hence exhibits the universal scaling laws in the universality class of the quantum Hall plateau transitions ${ }^{12,35}$. Free from any interaction, this single-Dirac-cone surface state is an ideal platform for studying the critical phenomena of 'metal-insulator' transitions in Dirac systems ${ }^{14,36}$.

In $2 \mathrm{D}$ photonic crystals, topological band structures protected by $\varepsilon-\mu$ symmetry ${ }^{37,38}$ have been studied. However, symmetries in constitutive relations are difficult to maintain over a wide frequency bandwidth. Another 2D example discusses the bulk topology of $C_{6}$ rotation $^{39}$. Unfortunately, six-fold rotation cannot be preserved on 
the $1 \mathrm{D}$ edge and cannot protect edge states. In contrast, our glide reflection can be maintained for all materials at all frequencies with protected surface states.

Experimentally, the $\mathcal{T}$-breaking BPI photonic crystals can be readily realized at microwave frequencies by assembling ferrimagnetic $\operatorname{rods}^{17,18}$ with internal remnant magnetization, without the need for external magnetic fields. Towards optical frequencies, $\mathcal{T}$-breaking could potentially be implemented through dynamic Floquet modulations ${ }^{40,41}$. In addition, our approach for photons can be used directly for phonons, where $\mathcal{T}$-breaking can be achieved by spinning the rods ${ }^{42}$.

This work demonstrates that symmetry-protected 3D topological bandgaps supporting disorder-immune surface states can be obtained in bosonic systems. Spatial symmetries ${ }^{2,8,12,43-45}$ (C. Fang et al., manuscript in preparation) other than the glide reflection are to be studied in the rich context of 230 space groups and 1651 magnetic groups for any bosonic particles.

\section{Received 22 July 2015; accepted 19 November 2015;} published online 4 January 2016

\section{References}

1. Thouless, D. J., Kohmoto, M., Nightingale, M. P. \& den Nijs, M. Quantized Hall conductance in a two-dimensional periodic potential. Phys. Rev. Lett. 49, 405-408 (1982).

2. Chiu, C.-K., Teo, J. C. Y., Schnyder, A. P \& Ryu, S. Classification of topological quantum matter with symmetries. Preprint at http://arXiv.org/abs/ 1505.03535 (2015).

3. Hasan, M. \& Kane, C. Colloquium: Topological insulators. Rev. Mod. Phys. 82, 3045-3067 (2010).

4. Qi, X.-L. \& Zhang, S.-C. Topological insulators and superconductors. Rev. Mod. Phys. 83, 1057-1110 (2011).

5. Zhang, H. et al. Topological insulators in $\mathrm{Bi}_{2} \mathrm{Se}_{3}, \mathrm{Bi}_{2} \mathrm{Te}_{3}$ and $\mathrm{Sb}_{2} \mathrm{Te}_{3}$ with a single Dirac cone on the surface. Nature Phys. 5, 438-442 (2009).

6. Moore, J. E. The birth of topological insulators. Nature 464, 194-198 (2010).

7. Lu, L., Joannopoulos, J. D. \& Soljačić, M. Topological photonics. Nature Photon. 8, 821-829 (2014).

8. Fu, L. Topological crystalline insulators. Phys. Rev. Lett. 106, 106802 (2011).

9. Hsieh, T. H. et al. Topological crystalline insulators in the SnTe material class. Nature Commun. 3, 982 (2012).

10. Ando, Y. \& Fu, L. Topological crystalline insulators and topological superconductors: From concepts to materials. Annu. Rev. Condens. Matter Phys. 6, 361-381 (2015).

11. Liu, C.-X., Zhang, R.-X. \& VanLeeuwen, B. K. Topological nonsymmorphic crystalline insulators. Phys. Rev. B 90, 085304 (2014)

12. Fang, C. \& Fu, L. New classes of three-dimensional topological crystalline insulators: Nonsymmorphic and magnetic. Phys. Rev. B 91, 161105 (2015).

13. Shiozaki, K., Sato, M. \& Gomi, K. Z 2 topology in nonsymmorphic crystalline insulators: Möbius twist in surface states. Phys. Rev. B 91, 155120 (2015).

14. Fu, L. \& Kane, C. L. Topology, delocalization via average symmetry and the symplectic Anderson transition. Phys. Rev. Lett. 109, 246605 (2012).

15. Fulga, I. C., van Heck, B., Edge, J. M. \& Akhmerov, A. R. Statistical topological insulators. Phys. Rev. B 89, 155424 (2014).

16. Taherinejad, M., Garrity, K. F. \& Vanderbilt, D. Wannier center sheets in topological insulators. Phys. Rev. B 89, 115102 (2014).

17. Wang, Z., Chong, Y., Joannopoulos, J. D. \& Soljačić, M. Observation of unidirectional backscattering-immune topological electromagnetic states. Nature 461, 772-775 (2009).

18. Skirlo, S. A., Lu, L., Igarashi, Y., Joannopoulos, J. \& Soljacic, M. Experimental observation of large chern numbers in photonic crystals. Preprint at http://arXiv.org/abs/1504.04399 (2015).

19. Varjas, D., de Juan, F. \& Lu, Y.-M. Bulk invariants and topological response in insulators and superconductors with nonsymmorphic symmetries. Phys. Rev. B 92, 195116 (2015).

20. Meiboom, S., Sammon, M. \& Berreman, D. W. Lattice symmetry of the cholesteric blue phases. Phys. Rev. A 28, 3553-3560 (1983).

21. Young, S. M. et al. Dirac semimetal in three dimensions. Phys. Rev. Lett. 108, 140405 (2012).

22. Liu, Z. K. et al. Discovery of a three-dimensional topological Dirac semimetal, $\mathrm{Na}_{3}$ Bi. Science 343, 864-867 (2014).

23. Wang, Z., Weng, H., Wu, Q., Dai, X. \& Fang, Z. Three-dimensional Dirac semimetal and quantum transport in $\mathrm{Cd}_{3} \mathrm{As}_{2}$. Phys. Rev. B 88, 125427 (2013).
24. Lu, L., Fu, L., Joannopoulos, J. D. \& Soljačić, M. Weyl points and line nodes in gyroid photonic crystals. Nature Photon. 7, 294-299 (2013).

25. Lu, L. et al. Experimental observation of Weyl points. Science 349, 622-624 (2015)

26. Mock, A., Lu, L. \& O’Brien, J. Space group theory and Fourier space analysis of two-dimensional photonic crystal waveguides. Phys. Rev. B 81, 155115 (2010).

27. Lu, L. et al. Three-dimensional photonic crystals by large-area membrane stacking. Opt. Lett. 37, 4726-4728 (2012)

28. Parameswaran, S. A., Turner, A. M., Arovas, D. P. \& Vishwanath, A. Topological order and absence of band insulators at integer filling in non-symmorphic crystals. Nature Phys. 9, 299-303 (2013).

29. Roy, R. Space group symmetries and low lying excitations of many-body systems at integer fillings. Preprint at http://arXiv.org/abs/1212.2944 (2012).

30. Young, S. M. \& Kane, C. L. Dirac semimetals in two dimensions. Phys. Rev. Lett. 115, 126803 (2015).

31. Watanabe, H., Po, H. C., Vishwanath, A. \& Zaletel, M. Filling constraints for spin-orbit coupled insulators in symmorphic and nonsymmorphic crystals. Proc. Natl Acad. Sci. USA 112, 14551-14556 (2015).

32. Haldane, F. D. M. \& Raghu, S. Possible realization of directional optical waveguides in photonic crystals with broken time-reversal symmetry. Phys. Rev. Lett. 100, 013904 (2008).

33. Yu, R., Qi, X. L., Bernevig, A., Fang, Z. \& Dai, X. Equivalent expression of $Z_{2}$ topological invariant for band insulators using the non-abelian Berry connection. Phys. Rev. B 84, 075119 (2011).

34. Alexandradinata, A., Dai, X. \& Bernevig, B. A. Wilson-loop characterization of inversion-symmetric topological insulators. Phys. Rev. B 89, $155114(2014)$

35. Ludwig, A. W. W., Fisher, M. P. A., Shankar, R. \& Grinstein, G. Integer quantum Hall transition: An alternative approach and exact results. Phys. Rev. B 50, 7526-7552 (1994).

36. Bardarson, J. H., Tworzydło, J., Brouwer, P. W. \& Beenakker, C. W. J. One-parameter scaling at the Dirac point in graphene. Phys. Rev. Lett. 99, 106801 (2007).

37. Khanikaev, A. B. et al. Photonic topological insulators. Nature Mater. 12, 233-239 (2013)

38. Chen, W.-J. et al. Experimental realization of photonic topological insulator in a uniaxial metacrystal waveguide. Nature Commun. 5, 5782 (2014).

39. Wu, L.-H. \& Hu, X. Scheme for achieving a topological photonic crystal by using dielectric material. Phys. Rev. Lett. 114, 223901 (2015).

40. Fang, K., Yu, Z. \& Fan, S. Realizing effective magnetic field for photons by controlling the phase of dynamic modulation. Nature Photon. 6, 782-787 (2012)

41. Rechtsman, M. C. et al. Photonic Floquet topological insulators. Nature 496, 196-200 (2013)

42. Wang, P., Lu, L. \& Bertoldi, K. Topological phononic crystals with one-way elastic edge waves. Phys. Rev. Lett. 115, 104302 (2015).

43. Liu, C.-X. Antiferromagnetic crystalline topological insulators. Preprint at http://arXiv.org/abs/1304.6455 (2013).

44. Alexandradinata, A., Fang, C., Gilbert, M. J. \& Bernevig, B. A. Spin-orbit-free topological insulators without time-reversal symmetry. Phys. Rev. Lett. 113, 116403 (2014).

45. Wang, Z., Alexandradinata, A., Cava, R. J. \& Bernevig, B. A. (in review, 2015).

\section{Acknowledgements}

We thank T. H. Hsieh, A. Alexandradinata, B. Andrei Bernevig, S. Skirlo, A. Men, J. Liu and F. Wang for discussions. S.G.J. and J.D.J. were supported in part by the US ARO. through the ISN, under Contract No. W911NF-13-D-0001. C.F. and L.F. were supported by the DOE Office of Basic Energy Sciences, Division of Materials Sciences and Engineering under Award No. DE-SC0010526. L.L. was supported in part by the MRSEC Program of the NSF under Award No. DMR-1419807. M.S. and L.L. (analysis and reading of the manuscript) were supported in part by the MIT S3TEC EFRC of DOE under Grant No. DE-SC0001299.

\section{Author contributions}

L.L. proposed the BP I structure and performed the calculations with the help of S.G.J. and C.F.; C.F. and L.F. conceived and analysed the band topology; all authors contributed to the discussion of the results and preparation of the manuscript.

\section{Additional information}

Supplementary information is available in the online version of the paper. Reprints and permissions information is available online at www.nature.com/reprints.

Correspondence and requests for materials should be addressed to L.L. or L.F.

\section{Competing financial interests}

The authors declare no competing financial interests. 